ФГБУ «Национальный медицинский исследовательский центр эндокринологии» Минздрава России, Москва, Россия

Обоснование: Вторичный остеопороз представляет собой значимую проблему, особенно у пациентов с эндокринной патологией, которая нередко не сопровождается яркой клинической симптоматикой. Необходимы маркеры костного происхождения, которые можно было бы использовать при установке диагноза остеопороза для уточнения его генеза, особенно у лиц молодого возраста, чаще имеющих вторичный остеопороз, нежели пожилые пациенты. При болезни Иценко-Кушинга (БИК) таким маркером, помимо остеокальцина, мог бы стать и другой маркер костеобразования - N-концевой пропептид проколлагена 1 типа (P1NP).

Цель: Изучить диагностические возможности Р1NP в качестве дополнительного маркера эндогенного гиперкортицизма (болезни Иценко-Кушинга) по сравнению с остеокальцином.

Mетоды: В исследование включены пациенты с болезнью Иценко-Кушинга и здоровые добровольцы, подобранные по полу, возрасту и индексу массы тела (ИМТ). Исследованы уровни остеокальцина и Р1NP в обеих группах, использован электрохемилюминесцентный метод: для P1NP на анализаторе Cobas e411, для остеокальцина - Cobas 6000 Module e601 (оба Roche, Швейцария). С целью определения отрезной точки для Р1NP в целях диагностики БИК осуществлен ROC-анализ с определением чувствительности и специфичности метода.

Результаты: Количество пациентов с болезнью Иценко-Кушинга составило 29 человек, здоровых лиц из контрольной группы - 27. Различий по возрасту, полу и ИМТ между ними не выявлено ( $p=0,488,0,426$ и 0,531 соответственно). Оба исследованных маркера костеобразования (остеокальцин и Р1NP) были снижены у пациентов с БИК: 8,53 нг/мл (Q25\%;Q75\% 5,40; 12,41) против 22,45 нг/мл (Q25\%;Q75\% 17,36; 26,31) (p <0,001) и 28,50 нг/мл (Q25\%;Q75\% 18,00; 44,00) против 56,50 нг/мл (Q25\%;Q75\% 39,50; 65,50) (p <0,001) соответственно. Площадь под кривой операционной характеристики (AUC) составила: для P1NP - 0,808 (95\% ДИ 0,693-0,924), для остеокальцина - 0,925 (95\% ДИ 0,857-0,992), что свидетельствует о большей диагностической ценности остеокальцина для верификации БИК среди здорового контроля. Получены оптимальные точки вмешательства: для P1NP - 53,4 нг/мл (значения ниже ее свойственны пациентам с БИК; чувствительность метода - 96,55\%, специфичность - 57,69\%), для остеокальцина - 15,285 нг/мл (ниже у пациентов с БИК; чувствительность составила 92,59\%, специфичность - 77,78\%).

Заключение: Диагностические возможности остеокальцина для выявления болезни Иценко-Кушинга в популяции выше в сравнении с P1NP. Тем не менее, применение P1NP может быть полезно, т.к., в отличие от остеокальцина, он является прямым показателем формирования коллагеновых структур костного матрикса, что важно для оценки степени угнетения синтеза коллагена 1 типа при БИК и ухудшения качества костной ткани вследствие глюкокортикоид-индуцированного остеопороза.

КЛЮЧЕВЫЕ СЛОВА: N-концевой пропептид проколлагена 1 типа (P1NP); остеокальцин; маркеры костного ремоделирования; вторичный остеопороз; болезнь Иценко-Кушинга.

\title{
EVALUATION OF DIAGNOSTIC POTENTIAL OF THE COLLAGEN OSTEOGENESIS MARKER (P1NP) COMPARED WITH OSTEOCALCIN IN CUSHING'S DISEASE
}

๑ Timur T. Tsoriev, Zhanna E. Belaya, Lyudmila Y. Rozhinskaya, Larisa V. Nikankina

Endocrinology Research Centre, Moscow, Russia

Background: Secondary osteoporosis is a significant problem, especially in patients with endocrine pathology, which is not accompanied constantly by distinct clinical symptoms. Markers of bone origin are needed, which could be used in osteoporosis diagnosis to clarify its genesis, especially in young people who have secondary osteoporosis more often than older patients. In Cushing's disease (CD), such a marker, in addition to osteocalcin, could be another bone formation marker, procollagen type $1 \mathrm{~N}$-terminal propeptide (P1NP).

Aims: To study the diagnostic potential of P1NP as an additional marker of endogenous hypercortisolism (Cushing's disease) compared to osteocalcin.

Materials and methods: The study involved patients with Cushing's disease and healthy volunteers, matched by gender, age, and body mass index. The levels of osteocalcin and P1NP were assessed in both groups, the electrochemiluminescence method for P1NP (Cobas e411 (Roche, Switzerland)) and for osteocalcin (Cobas 6000 Module e601 (Roche, Switzerland)) was used. ROC analysis was performed with the calculation of sensitivity and specificity of the method to determine the cut-off point for P1NP in CD diagnosis. 
Results: 29 patients with Cushing's disease and 27 healthy individuals from the control group were included in the study. There were no differences in age, sex and body mass index ( $p=0.488,0.426$ and 0.531 , respectively). Both studied bone formation markers (osteocalcin and P1NP) were reduced in patients with CD: $8.53 \mathrm{ng} / \mathrm{ml}$ (Q25\%;Q75\% 5.40; 12.41) versus $22.45 \mathrm{ng} / \mathrm{ml}$ (Q25\%;Q75\% 17.36; 26.31) ( $\mathrm{p}<0.001)$ and $28.50 \mathrm{ng} / \mathrm{ml}(\mathrm{Q} 25 \% ; \mathrm{Q} 75 \% 18.00 ; 44.00)$ versus $56.50 \mathrm{ng} / \mathrm{ml}(\mathrm{Q} 25 \% ; \mathrm{Q} 75 \%$ 39.50; $65.50)(p<0.001)$, respectively. The area under the receiver operating characteristic curve (AUC) was $0.808(95 \% \mathrm{Cl} 0.693-$ $0.924)$ for P1NP and 0.925 (95\% Cl 0.857-0.992) for osteocalcin, that indicates the greater diagnostic value of osteocalcin for CD verification in healthy controls. Optimal cut-off points were obtained: $53.4 \mathrm{ng} / \mathrm{ml}$ (values below are more typical for patients with CD; sensitivity of the method is $96.55 \%$, specificity $57.69 \%$ ) for P1NP and $15.285 \mathrm{ng} / \mathrm{ml}$ (below for patients with CD; sensitivity was $92.59 \%$, specificity $77.78 \%$ ) for osteocalcin.

Conclusions: The diagnostic potential of osteocalcin to detect Cushing's disease in the population is higher compared to P1NP. However, applying of P1NP can be useful because, unlike osteocalcin, it is a direct indicator of the formation of bone matrix collagen structures, that is important for assessing the degree of inhibition of collagen type 1 synthesis in CD and deterioration of bone tissue due to glucocorticoid-induced osteoporosis.

KEYWORDS: P1NP; osteocalcin; bone remodeling; osteoporosis; pituitary ACTH hypersecretion.

\section{ОБОСНОВАНИЕ}

Эндогенный гиперкортицизм, и в частности болезнь Иценко-Кушинга (БИК), представляет собой тяжелое хроническое эндокринное заболевание, основой патогенеза которого является гиперпродукция надпочечниками кортизола, что приводит к усилению катаболических процессов практически во всех тканях организма и, как следствие, полиорганным нарушениям [1]. Одним из них является глюкокортикоид-индуцированный остеопороз (ГКО), который характеризуется снижением костеобразования под действием гиперкортизолемии и приводит в конечном итоге к низкотравматическим переломам [2, 3].

В связи с тем, что болезнь Иценко-Кушинга развивается у пациентов достаточно молодого, т.е. трудоспособного, возраста [4, 5], ГКО (даже без учета других осложнений гиперкортицизма) представляется весьма значимой проблемой не только медицинского, но и социально-экономического характера. Его диагностика с помощью стандартной методики - рентгеновской остеоденситометрии - нередко затруднена: низкотравматические переломы при ГКО могут происходить при значениях минеральной плотности кости, соответствующих остеопении и даже возрастной норме [6-8], что указывает на недостаточную чувствительность метода при данной патологии. Необходим вспомогательный маркер, специфичный для костной ткани, который бы позволял заподозрить наличие ГКО и в целом болезни Иценко-Кушинга. Ранее таким маркером был назван остеокальцин [9], однако он относится к неколлагеновым белкам костного матрикса и поэтому лишь отчасти может отражать деструкцию костной структуры, особенно трабекулярной. В качестве альтернативы нами решено было рассмотреть маркер коллагеновой природы - P1NP.

$\mathrm{N}$-концевой пропептид проколлагена 1 типа (procollagen type $1 \mathrm{~N}$-terminal propeptide, P1NP) является специфическим продуктом остеобластов и в меньшей степени - фибробластов, протеолитически отщепляясь от молекулы проколлагена 1 типа. Его сывороточный уровень, таким образом, прямо коррелирует с концентрацией коллагена 1 типа в костной ткани. Высвобождаясь в кровоток в виде трехмерной структуры (интактный P1NP), он уже при температуре $37^{\circ} \mathrm{C}$ распадается с образованием низкомолекулярной (мономерной) формы, стабильной как in vivo [10], так и in vitro при комнатной температуре [11]. Преимущественно мономерная форма обнаруживается в сыворотке крови пациентов с терминальной почечной недостаточностью (ХБП С5Д) и ограничением подвижности (пожилые лежачие больные) [12]. Это объясняется особенностями клиренса этого соединения: интактная форма метаболизируется в основном печенью, в то время как мономерная выводится почками, что затрудняет определение истинных значений маркера при использовании диагностических наборов, измеряющих уровень общего P1NP (включающего обе формы) [13]. В проведенных ранее исследованиях Р1NP продемонстрировал низкую индивидуальную вариабельность [14] и отсутствие ярко выраженного циркадианного характера выделения в кровь [15]. Он успел зарекомендовать себя при постменопаузальном остеопорозе в качестве чувствительного маркера эффективности лечения при назначении анаболической терапии: его содержание в крови увеличивалось в течение первых 3 месяцев на $40-80 \%$ и более $[15,16]$. Благодаря этому P1NP был рекомендован для использования в клинических исследованиях при мониторинге эффективности лечения остеопороза Международным фондом по изучению остеопороза (IOF) и Международной федерацией клинической химии и лабораторной медицины (IFCC) [14].

\section{ЦЕЛЬ}

Изучить диагностические возможности P1NP в качестве дополнительного маркера эндогенного гиперкортицизма (болезни Иценко-Кушинга) по сравнению с остеокальцином.

\section{МЕТОДЫ}

\section{Дизайн исследования}

Исследование проведено как одноцентровое одномоментное по типу «случай-контроль», со сплошной выборкой пациентов (при условии соответствия критериям включения).

\section{Критерии соответствия}

Критерии включения: установленный согласно действующим клиническим рекомендациям [17] диагноз БИК в активной стадии заболевания (до нейрохирурги- 
ческого лечения). Здоровые добровольцы для формирования группы контроля подбирались по полу, возрасту и индексу массы тела (ИМТ).

Критериями исключения являлись: ремиссия заболевания; прием глюкокортикоидов (в т.ч. вследствие надпочечниковой недостаточности в исходе хирургического лечения), медикаментов, оказывающих значимое модифицирующее влияние на минеральный и костный обмен (препараты тестостерона, менопаузальная гормональная терапия, комбинированные оральные контрацептивы, экзогенный гормон роста), и препаратов для лечения остеопороза на момент включения в исследование; первичный и вторичный гипогонадизм, в т.ч. требующий приема половых стероидов; гипопитуитаризм; тяжелые, жизнеугрожающие состояния (острая почечная и печеночная недостаточность, инфаркт миокарда, острое нарушение мозгового кровообращения и пр.) и терминальные состояния; острые инфекции; обострение хронических заболеваний; тяжелые психиатрические заболевания; длительная (>1 недели) общая иммобилизация; беременность; злоупотребление алкоголем; другие заболевания, приводящие к вторичному остеопорозу (помимо эндогенного гиперкортицизма).

\section{Условия проведения}

Исследование проведено на базе отделения нейроэндокринологии и остеопатий ФГБУ «Национальный медицинский исследовательский центр эндокринологии» МЗ РФ (г. Москва). Включались пациенты с клинически и лабораторно подтвержденным диагнозом болезни Иценко-Кушинга, находившиеся на обследовании в указанном отделении, а также здоровые лица, вошедшие в контрольную группу. Каких-либо специфических факторов, способных повлиять на внешнюю обобщаемость выводов исследования и возможность ихэкстраполяции, не выявлено. Все участники - как пациенты, так и здоровые лица - подписали информированное согласие.

\section{Продолжительность исследования}

Набор материала продолжался с осени 2015 г. по осень 2016 г. Изучаемые маркеры костеобразования исследовались в сыворотке крови одномоментно после завершения сбора биологических образцов.

\section{Описание медицинского вмешательства}

У всех участников исследования однократно производился забор крови натощак с последующим центрифугированием при температуре $-5^{\circ} \mathrm{C}$ в течение 30 мин (с частотой 3000 об./мин) и замораживанием взятых образцов в аликвотах при температуре ниже $-20^{\circ} \mathrm{C}$. Предварительно участники заполняли опросники для выяснения их соответствия критериям включения/исключения (см. Критерии соответствия).

\section{Основной исход исследования}

Точки вмешательства (отрезные точки) для маркеров костеобразования - остеокальцина и P1NP, - а также их чувствительность и специфичность были рассмотрены в качестве суррогатных конечных точек нашего исследования. Данные показатели позволяют оценить применимость P1NP в качестве дополнительного диагностического маркера при болезни Иценко-Кушинга.

\section{Дополнительные исходы исследования}

Показатели гормональной активности аденомы гипофиза у пациентов с БИК: значения кортизола и АКТГ в утреннее (8:00) и вечернее (23:00) время, свободного кортизола в суточной моче и кортизола в вечерней (23:00) слюне.

\section{Методы регистрации исходов}

Для исследования маркеров костеобразования применялся электрохемилюминесцентный метод: Cobas 6000 Module e601 (Roche, Швейцария) - для остеокальцина (референсный интервал 11-43 нг/мл) и Cobas e411 (Roche, Швейцария) - для P1NP. Референсные значения последнего зависят от возраста и пола пациента [18]:

\section{- Для мужчин:}

моложе 1 года: 830-1850 нг/мл;

1-2,9 года: 400-1500 нг/мл;

3-11,9 лет: 300-900 нг/мл;

12-16,9 лет: 220-1600 нг/мл;

17-17,9 лет: 80-480 нг/мл;

18-20,9 лет: 60-220 нг/мл;

21-24,9 лет: 35-140 нг/мл;

25 лет и старше: 20-85 нг/мл.

- Для женщин:

моложе 1 года: 900-1800 нг/мл;

1-2,9 года: 500-1500 нг/мл;

3-9,9 лет: 300-1000 нг/мл;

10-13,9 лет: 140-1200 нг/мл;

14-17,9 лет: 40-460 нг/мл;

18-24,9 лет: 20-110 нг/мл;

25-34,9 лет: 20-85 нг/мл;

35 лет и старше (пременопауза): 15-60 нг/мл;

35 лет и старше (постменопауза): 20-115 нг/мл.

Диагноз болезни Иценко-Кушинга устанавливался или исключался, исходя из действующих клинических рекомендаций [17], с определением суточного ритма кортизола и АКТГ, проведением анализа свободного кортизола в суточной моче и кортизола в вечерней слюне, МРТ головного мозга, а также, при необходимости дифференциальной диагностики причин АКТГ-зависимого гиперкортицизма -селективного забора венозной крови из нижних каменистых синусов. АКТГ в плазме и кортизол в сыворотке крови оценивались с помощью электрохемилюминесцентного метода (Cobas 6000 Module e601 (Roche, Швейцария)). Референсные значения составили: для кортизола 46-270 нмоль/л в вечерние часы; для АКТГ - 7-66 пг/мл утром и 0-30 пг/мл соответственно. Для определения уровня свободного кортизола в слюне использован электрохемилюминесцентный модуль Cobas e601 (Roche, Швейцария) (0,5-9,4 нмоль/л). Свободный кортизол в суточной моче измерен иммунохемилюминесцентным методом (анализатор Vitros ECi (США, Великобритания) (референсные значения 60-413 нмоль/сут)).

\section{Этическая экспертиза}

Локальным Этическим комитетом ФГБУ «Национальный медицинский исследовательский центр эндокринологии» Минздрава России, согласно протоколу №12 Заседания Комитета от 28.10.2015 г., постановлено, что планируемая научная работа соответствует этическим стандартам добросовестной клинической практики 
и может быть проведена на базе отделения нейроэндокринологии и остеопатий ФГБУ «НМИЦЭ» МЗ РФ.

Все пациенты подписывали добровольное информированное согласие на участие в исследовании.

\section{Статистический анализ}

Принципы расчета размера выборки: Ввиду отсутствия пилотных исследований, а также в силу редкой встречаемости болезни Иценко-Кушинга в популяции размер выборки был установлен эмпирически.

Методы статистического анализа данных: Анализ данных производился в пакете статистических программ IBM SPSS Statistics Base (SPSS, США). Нормальность распределения устанавливалась тестом Колмогорова-Смирнова. Количественные значения признаков представлены как медианы (median, Me) и 25-й и 75-й процентили (Q25\%;Q75\%). Для оценки различий по количественным параметрам был использован непараметрический вариант однофакторного дисперсионного анализа - критерий Манна-Уитни. Корреляционный анализ проводился также непараметрическим методом применялся расчет коэффициента корреляции Спирмена (р). С целью определения отрезной точки (cut-off point) диагностики БИК с использованием P1NP в качестве дополнительного маркера был осуществлен ROC-анализ (построение кривой операционной характеристики) с определением чувствительности и специфичности метода. В качестве оптимальной отрезной точки был принят уровень показателя, которому соответствовало максимальное значение суммы чувствительности (пропорция истинно положительных результатов, подтвержденных тестируемым методом, по отношению к золотому стандарту) и специфичности метода (пропорция истинно отрицательных результатов, подтвержденных тестируемым методом, по отношению к золотому стандарту), полученных в ходе ROC-анализа [19]. Прогностическая ценность положительного результата (количество истинно положительных результатов диагностического теста / (количество истинно положительных результатов диагностического теста + количество ложноположительных результатов диагностического теста)); прогностическая ценность отрицательного результата (количество истинно отрицательных результатов диагностического теста / (количество истинно отрицательных результатов диагностического теста + количество ложноотрицательных результатов диагностического теста)); отношение правдоподобия для положительного результата теста (чувствительность / (1 - специфичность)) - были рассчитаны согласно указаниям, приведенным в специализированных изданиях [20] и тематических публикациях [21]. Площади под кривыми операционных характеристик (AUC) были измерены для демонстрации возможностей диагностических тестов верно оценивать истинно положительные и истинно отрицательные результаты и для сравнения качества этих тестов $[22,23]$. Расчет доверительных интервалов (ДИ) чувствительности и специфичности произведен с помощью онлайн-калькулятора Confidence Interval Calculator (https://www.pedro.org.au/ wp-content/uploads/Clcalculator/, последнее обновление 24.03.2011 г.), основанного на методе Уилсона [24].

\section{РЕЗУЛЬТАТЫ}

Объекты (участники) исследования

Было обследовано 29 пациентов с болезнью Иценко-Кушинга и 27 условно здоровых лиц, из них 22 мужчины и 34 женщины. Общая характеристика участников исследования приведена в Таблице 1. За основной диагностический признак эндогенного гиперкортицизма принят уровень кортизола в вечерней слюне (как достаточно точный показатель избыточной продукции кортизола, имеющий преимущество в виде более простой методики получения диагностического материала и большей комплаэнтности обследуемых при выполнении требований к правильности его сбора и при этом не уступающий по чувствительности определению суточной экскреции кортизола [25]).

Таблица 1. Краткая характеристика включенных в исследование лиц (приводится медиана и 25-75 процентили)

\begin{tabular}{|c|c|c|c|}
\hline \multirow{2}{*}{ Показатели } & \multicolumn{2}{|c|}{ Me (Q25;Q75) } & \multirow[t]{2}{*}{$\mathbf{p}$} \\
\hline & БИК & Контроль & \\
\hline Количество пациентов & 29 & 27 & \\
\hline Возраст, лет & $39,93(34,07 ; 45,79)$ & $34,69(30,32 ; 39,07)$ & 0,155 \\
\hline Пол (М\%:ж\%) & $9: 20$ (31,03\%:68,97\%) & $13: 14(48,15 \%: 51,85 \%)$ & 0,527 \\
\hline ИМТ, кг/м² & $26,57(22,61 ; 30,74)$ & $25,69(22,00 ; 30,66)$ & 0,771 \\
\hline $\begin{array}{l}\text { Кортизол в вечерней } \\
\text { слюне, нмоль/л }\end{array}$ & $20,56(12,18 ; 43,91)$ & $1,66(0,69 ; 3,92)$ & $<0,001$ \\
\hline Остеокальцин, нг/мл & $8,53(5,40 ; 12,41)$ & $22,45(17,36 ; 26,31)$ & $<0,001$ \\
\hline $\begin{array}{l}\text { С-концевой телопептид } \\
\text { коллагена } 1 \text { типа, нг/мл }\end{array}$ & $0,41(0,27 ; 0,53)$ & $0,43(0,33 ; 0,64)$ & 0,7 \\
\hline P1NP, нг/мл & $28,50(18,00 ; 44,00)$ & $56,50(39,50 ; 65,50)$ & $<0,001$ \\
\hline
\end{tabular}




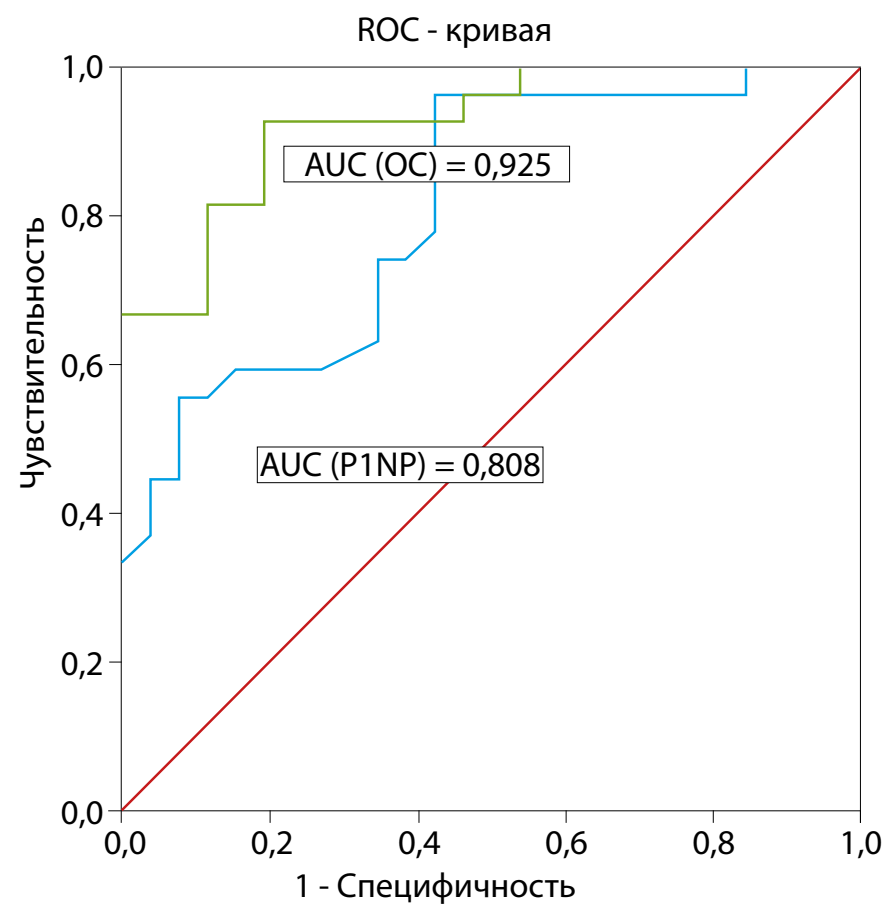

Diagonal segments are produced by ties

- P1NP — Остеокальцин — Линия референса

Рисунок 1. ROC-анализ для отрезных точек P1NP и остеокальцина при эндогенном гиперкортицизме (БИК)

\section{Основные результаты исследования}

Площадь под кривой операционной характеристики (AUC) была существенно меньше для P1NP - 0,808 (95\% ДИ 0,693-0,924) - в сравнении с остеокальцином - 0,925 (95\% ДИ 0,857-0,992) (см. Рисунок 1), что свидетельствует о большей диагностической ценности остеокальцина для верификации БИК среди здорового контроля. Однако при таком значении площади под кривой операционной характеристике P1NP также имеет значение для диагностики.

На основании ROC-анализа были получены различные точки вмешательства, из которых выбраны оптимальные (максимальная сумма чувствительности и специфичности) (см. Таблицу 2). Значения ниже отрезных точек (cutoff point) для обоих маркеров свойственны пациентам с БИК. Исходя из приведенных в таблице результатов, можно заключить, что, P1NP также может рассматриваться как потенциальный диагностический маркер при БИК.

\section{Дополнительные результаты исследования}

Результаты исследования свободного кортизола в суточной моче, как и данные анализа кортизола в вечерней слюне, подтвердили наличие эндогенного гиперкортицизма: медиана составила 1332,00 нмоль/сут (584,80;3850,00). Показатели АКТГ утром и вечером - 84,6 пг/мл $(64,22 ; 104,98)$ и 67,09 пг/мл $(51,37 ; 82,82)$ соответственно. Таким образом, уровни гормонов существенно превышают референсные значения для использованных диагностических наборов. У всех пациентов было подтверждено наличие аденомы гипофиза, ввиду чего диагноз болезни Иценко-Кушинга у включенных в эту группу не вызывает сомнений.

При корреляционном анализе выявлена отрицательная связь между уровнем кортизола в вечерней слюне и P1NP ( $\rho=-0,651, p<0,001)$, а также сильная положительная связь между остеокальцином и P1NP ( $\rho=0,915$, $\mathrm{p}<0,001)$.

\section{Нежелательные явления}

Нежелательных явлений в ходе исследования отмечено не было.

\section{ОБСУЖДЕНИЕ}

Резюме основного результата исследования

Было установлено подавление маркеров костеобразования - остеокальцина и $\mathrm{N}$-концевого пропептида проколлагена 1 типа (P1NP) у пациентов с болезнью Иценко-Кушинга. В результате ROC-анализа возможность использования Р1NP в диагностике БИК подтверждена, однако, несмотря на хорошую чувствительность маркера, по специфичности он значительно уступает остеокальцину, ранее уже зарекомендовавшему себя в качестве дополнительного диагностического теста при этом заболевании. Тем не менее, Р1NP может быть полезен для применения у пациентов с БИК и эндогенным гиперкортицизмом ввиду своего происхождения, благодаря

Таблица 2. Диагностические индексы для исследования остеокальцина и Р1NP в сыворотке крови в целях выявления эндогенного гиперкортицизма (БИК)

\begin{tabular}{|c|c|c|}
\hline \multirow{2}{*}{ Диагностические индексы } & \multicolumn{2}{|c|}{ Показатель (95\% ДИ) } \\
\hline & Остеокальцин & P1NP \\
\hline Отрезная точка (нг/мл) & 15,285 & 53,400 \\
\hline Чувствительность (\%) & $92,59(76,63-97,94)$ & $96,55(82,82-99,39)$ \\
\hline Специфичность (\%) & $77,78(59,24-89,39)$ & $57,69(38,95-74,46)$ \\
\hline $\begin{array}{l}\text { Прогностическая ценность } \\
\text { положительного результата }\end{array}$ & $4,167(2,041-8,506)$ & $2,282(1,449-3,594)$ \\
\hline $\begin{array}{l}\text { Прогностическая ценность } \\
\text { отрицательного результата }\end{array}$ & $0,095(0,025-0,367)$ & $0,060(0,008-0,422)$ \\
\hline $\begin{array}{l}\text { Отношение правдоподобия для } \\
\text { положительного результата теста }\end{array}$ & $43,750(7,975-240,018)$ & $38,182(4,488-324,856)$ \\
\hline
\end{tabular}


которому он способен давать представление об интенсивности процессов синтеза и деградации коллагенового матрикса.

\section{Обсуждение основного результата исследования}

У пациентов с болезнью Иценко-Кушинга, в отличие от лиц из группы контроля, маркеры костеобразования были подавлены: как ранее описанный остеокальцин $[26,27]$, так и маркер костеобразования коллагеновой природы - P1NP. Последний также может рассматриваться как потенциальный диагностический биомаркер эндогенного гиперкортицизма, но его диагностические возможности уступают таковым остеокальцина при прямом сравнении по площади под кривой операционной характеристики. При этом известно, что остеокальцин в декарбоксилированной форме у грызунов и потенциально - у человека обладает гормональной активностью, участвуя в регуляции углеводного обмена, увеличивая секрецию инсулина и улучшая выживаемость $\beta$-клеток поджелудочной железы $[28,29]$. Может ли это обстоятельство влиять на уровень остеокальцина в сыворотке крови, а также обусловливать бо́льшую чувствительность этого маркера при БИК по сравнению с Р1NP, остается предметом для дальнейшего изучения.

Как и ранее с остеокальцином [26], обнаружена отрицательная корреляция между Р1NP и кортизолом в вечерней слюне. Это в очередной раз подтверждает подавление костеобразования при эндогенном гиперкортицизме, в частности при БИК. При этом очень сильна взаимосвязь остеокальцина и P1NP. Следовательно, в качестве маркера костеобразования Р1NP может использоваться в клинической практике у пациентов с БИК наравне с остеокальцином. Несмотря на меньшую специфичность определения Р1NP в целях диагностики БИК, этот биомаркер является белком коллагеновой природы и непосредственным индикатором формирования коллагена 1 типа, составляющего основу органического матрикса костной ткани, а стало быть, способен точнее отражать процессы, происходящие в трабекулярном компартменте кости в условиях эндогенного гиперкортицизма. Кроме того, он не обладает, по имеющимся на сегодняшний день данным, гормональной активностью, что в определенных случаях также может давать ему преимущество перед остеокальцином.

Клинические ситуации, при которых измерение P1NP нерационально ввиду ложно завышенных значений терминальная почечная недостаточность (ХБП С5Д) [13] и метастатическое поражение костей [30], - были исключены в нашем исследовании еще на этапе формирования групп, поэтому сомнений в достоверности полученных результатов нет.

\section{Ограничения исследования}

На полученные в нашем исследовании результаты мог оказать влияние малый объем произведенной выборки (каждая из рассмотренных групп включала в себя не более 30 человек). Однако подобное исследование (оценка диагностических возможностей P1NP для выявления БИК в популяции) проводится впервые, поэтому эмпирическое определение количества участников в группах могло иметь место. Кроме того, редкость данного заболевания оказывала влияние на формирование выборки (осуществление сплошного набора участников по принципу соответствия критериям включения).

\section{ЗАКЛЮЧЕНИЕ}

Подавление уровня маркеров костеобразования остеокальцина и P1NP - при болезни Иценко-Кушинга отражает угнетение синтеза костного матрикса вследствие гиперкортицизма. У лабораторных животных (в частности, у мышей) уже было доказано наличие гормональной активности декарбоксилированной формы остеокальцина, в то время как эндо- или паракринные эффекты P1NP установлены не были. Вовлеченность остеокальцина в метаболические процессы у человека, степень взаимосвязи нарушений углеводного обмена, часто развивающихся у пациентов с болезнью Иценко-Кушинга, с бо́льшей диагностической («разрешающей») способностью измерения остеокальцина по сравнению с P1NP в этой когорте больных недостаточно изучена и требует уточнений. Проведенное нами исследование позволяет сделать вывод о преимуществе остеокальцина над P1NP при их использовании в качестве диагностических тестов с целью выявления болезни Иценко-Кушинга в популяции, несмотря на сильную корреляцию между этими маркерами и значимую отрицательную связь между активностью заболевания и уровнем P1NP. Могут ли нарушение метаболизма глюкозы, гиперинсулинемия и инсулинорезистентность влиять на уровень остеокальцина в сыворотке крови, а также обусловливать бо́льшую чувствительность этого маркера при БИК по сравнению с P1NP, на сегодняшний день неизвестно. Использование остеокальцина в качестве дополнительного диагностического критерия в случаях подозрения на вторичный характер остеопороза, особенно у молодых пациентов, в целом представляется оправданным. Что касается P1NP, то, учитывая тесную связь сывороточной концентрации этого показателя с интенсивностью синтеза коллагена 1 типа, может иметь смысл его применение в целях диагностики и контроля эффективности лечения глюкокортикоид-индуцированного остеопороза, что, однако, требует дальнейших исследований на эту тему.

\section{ДОПОЛНИТЕЛЬНАЯ ИНФОРМАЦИЯ.}

Источник финансирования. Исследование выполнено при финансовой поддержке Гранта Президента РФ для молодых ученых МД3204.2017.7.

Конфликт интересов. Авторы декларируют отсутствие явных и потенциальных конфликтов интересов, связанных с публикацией настоящей статьи.

Участие авторов. Т.Т. Цориев - планирование исследования, обзор литературы по теме исследования, набор участников групп (пациенты и контроль) согласно критериям соответствия, сбор клинического материала (физикальные, лабораторные данные), проведение статистического анализа полученных данных, написание текста статьи; Ж.Е. Белая - планирование исследования, руководство проведением исследования, проведение статистического анализа полученных данных, редактирование текста статьи; Л.я. Рожинская - научное консультирование в ходе написания статьи в качестве эксперта; Л.В. Никанкина - проведение анализа лабораторных показателей (остеокальцина и Р1NP) в предоставленных образцах сыворотки крови. Авторы прочли и одобрили финальную версию статьи перед публикацией. 


\section{СПИСОК ЛИТЕРАТУРЫ | REFERENCES}

1. Марова Е.И., Арапова С.Д., Белая Ж.Е., и др. Болезнь ИценкоКушинга: клиника, диагностика, лечение. - М.: ГЭОТАР-Медиа, 2012. [Marova El, Arapova SD, Belaya ZE, et al. Bolezn'Itsenko-Kushinga: klinika, diagnostika, lechenie. Moscow: GEOTAR-Media; 2012. (In Russ).]

2. Драгунова Н.В., Белая Ж.Е., Рожинская Л.Я. Состояние костномышечной системы при эндогенном гиперкортицизме. // Остеопороз и остеопатии. - 2012. - Т. 15. - №3. - С. 18-24. [Dragunova NV, Belaya ZE, Rozhinskaya LY. Musculoskeletal System in the Endogenous Hypercortisolism. OSTEO. 2012;15(3):18-24. (In Russ).] doi: https://doi.org/10.14341/osteo2012318-24.

3. Белая Ж.Е., Драгунова Н.В., Рожинская Л.Я., и др. Низкотравматичные переломы у пациентов с эндогенным гиперкортицизмом. Предикторы и факторы риска, влияние на качество жизни. // Остеопороз и остеопатии. - 2013. - Т. 16. - №1. - C. 7-13. [Belaya ZE, Dragunova NV, Rozhinskaya LY, et al. Low-Traumatic Fractures in Patients with Endogenous Hypercortisolism. Predictors and Risk Factors, the Impact on Quality of Life. OSTEO. 2013;16(1):7-13. (In Russ).] doi: https://doi.org/10.14341/osteo201317-13.

4. Lacroix A, Feelders RA, Stratakis CA, Nieman LK. Cushing's syndrome. The Lancet. 2015;386(9996):913-927. doi: https://doi. org/10.1016/s0140-6736(14)61375-1

5. Burton T, Le Nestour E, Neary M, et al. Algorithm development and the clinical and economic burden of Cushing's disease in a large US health plan database. Pituitary. 2016;19(2):167-174. doi: https://doi. org/10.1007/s11102-015-0695-9.

6. Eller-Vainicher C, Morelli V, Ulivieri FM, et al. Bone quality, as measured by trabecular bone score in patients with adrenal incidentalomas with and without subclinical hypercortisolism. J Bone Miner Res. 2012;27(10):2223-2230. doi: https://doi.org/10.1002/jbmr.1648.

7. Belaya ZE, Hans D, Rozhinskaya LY, et al. The risk factors for fractures and trabecular bone-score value in patients with endogenous Cushing's syndrome. Arch Osteoporos. 2015;10:44 doi: https://doi.org/10.1007/s11657-015-0244-1.

8. Vinolas H, Grouthier V, Mehsen-Cetre N, et al. Assessment of vertebral microarchitecture in overt and mild Cushing's syndrome using trabecular bone score. Clin Endocrinol (Oxf). 2018;89(2):148-154. doi: https://doi.org/10.1111/cen.13743.

9. Белая Ж.Е., Рожинская Л.Я., Мельниченко Г.А., и др. Возможности маркера костного обмена - остеокальцина - для диагностики эндогенного гиперкортицизма и вторичного остеопороза. // Остеопороз и остеопатии. - 2011. - Т.14. - №2. - С. 7-10. [Belaya ZE, Rozhinskaya LY, Mel'nichenko GA, et al. Vozmozhnosti markera kostnogo obmena - osteokal'tsina - dlya diagnostiki endogennogo giperkortitsizma i vtorichnogo osteoporoza. OSTEO. 2011;14(2):7-10. (In Russ).] doi: https://doi.org/10.14341/ osteo201127-10

10. Brandt J, Krogh TN, Jensen $\mathrm{CH}$, et al. Thermal instability of the trimeric structure of the $\mathrm{N}$-terminal propeptide of human procollagen type I in relation to assay technology. Clin Chem. 1999:45(1):47-53.

11. Stokes FJ, Ivanov P, Bailey LM, et al. The effects of sampling procedures and storage conditions on short-term stability of blood-based biochemical markers of bone metabolism. Clin Chem. 2011;57(1):138-140. doi: https://doi.org/10.1373/ clinchem.2010.157289.

12. Song L. Calcium and bone metabolism indices. Adv Clin Chem. 2017:82:1-46. doi: https://doi.org/10.1016/bs.acc.2017.06.005.

13. Koivula M-K, Risteli L, Risteli J. Measurement of aminoterminal propeptide of type I procollagen (PINP) in serum. Clin Biochem. 2012;45(12):920-927. doi: https://doi.org/10.1016/j.clinbiochem.2012.03.023

14. Vasikaran S, Eastell R, Bruyère O, et al. for the IOF-IFCC Bone Marker Standards Working Group. Markers of bone turnover for the prediction of fracture risk and monitoring of osteoporosis treatment: a need for international reference standards. Osteoporos Int. 2011;22(2):391-420. doi: https://doi.org/10.1007/s00198-0101501-1.

15. Brown JP, Albert C, Nassar BA, et al. Bone turnover markers in the management of osteoporosis. Clin Biochem. 2009;42(10-11):929942. doi: https://doi.org/10.1016/j.clinbiochem.2009.04.001.
16. Hlaing TT, Compston JE. Biochemical markers of bone turnover uses and limitations. Ann. Clin. Biochem. 2014;51(2):189-202. doi: https://doi.org/10.1177/0004563213515190

17. Мельниченко Г.А., Дедов И.И., Белая Ж.Е., и др. Болезнь Иценко-Кушинга: клиника, диагностика, дифференциальная диагностика, методы лечения. // Проблемы эндокринологии. 2015. - T.61. - №2. - C. 55-77. [Melnichenko GA, Dedov II, Belaya ZE, et al. Cushing's disease: the clinical features, diagnostics, differential diagnostics, and methods of treatment. Problems of Endocrinology. 2015;61(2):55. (In Russ).] doi: https://doi.org/10.14341/ probl201561255-77.

18. Labnet.health.nz [Internet]. Christchurch, New Zealand: Canterbury Health Laboratories; c2013-2019 [cited 2019 Jun 24]. Available from: http://www.labnet.health.nz/testmanager/index.php?fuseaction=main.DisplayTest\&testid $=1433$.

19. Zou KH, O'Malley AJ, Mauri L. Receiver-operating characteristic analysis for evaluating diagnostic tests and predictive models. Circulation. 2007;1 15(5):654-657. doi: https://doi.org/10.1161/circulationaha.105.594929.

20. Kramer MS. Clinical epidemiology and biostatistics: A primer for clinical investigators and decision-makers. 1st ed. Berlin: Springer-Verlag Berlin Heidelberg; 1988. 201-219 pp.

21. Hajian-Tilaki K. Receiver Operating Characteristic (ROC) Curve Analysis for Medical Diagnostic Test Evaluation. Caspian J Intern Med. 2013;4(2):627-635.

22. Hanley JA, MCNeil BJ. The meaning and use of the area under a receiver operating characteristic (ROC) curve. Radiology. 1982;143(1):29-36. doi: https://doi.org/10.1148/radiology.143.1.7063747

23. Hanley JA, MCNeil BJ. A method of comparing the areas under receiver operating characteristic curves derived from the same cases. Radiology. 1983;148(3):839-843. doi: https://doi.org/10.1148/ radiology.148.3.6878708.

24. Newcombe RG. Two-sided confidence intervals for the single proportion: comparison of seven methods. Stat. Med. 1998;17(8):857-872. doi: https://doi.org/10.1002/(sici)10970258(19980430)17:8<857::aid-sim777>3.0.co;2-e

25. Белая Ж.Е., Ильин А.В., Мельниченко Г.А., и др. Автоматизированный электрохемилюминесцентный метод определения кортизола в слюне для диагностики эндогенного гиперкортицизма среди пациентов с ожирением. // Ожирение и метаболизм. - 2011. - T. 8. - №2. - C. 56-63. [Belaya ZE, Il'in AV, Mel'nichenko GA, et al. Avtomatizirovannyy elektrokhemilyuminestsentnyy metod opredeleniya kortizola v slyune dlya diagnostiki endogennogo giperkortitsizma sredi patsientov s ozhireniem. Obesity and metabolism. 2011; (2):56-63. (In Russ).] doi: https://doi.org/10.14341/2071-8713-4954.

26. Белая Ж.Е., Рожинская Л.Я., Драгунова Н.В., и др. Сывороточные концентрации белков регуляторов остеобластогенеза и остеокластогенеза у пациентов с эндогенным гиперкортицизмом. // Остеопороз и остеопатии. - 2012. - Т.15. - №2. - C. 3-8. [Belaya ZE, Rozhinskaya LY, Dragunova NV, et al. Serum Concentrations of Protein Regulators Osteoblastogenesis and Osteoclastogenesis in Patients with Endogenous Hypercorticism. OSTEO. 2012;15(2):3-8. (In Russ).] doi: https://doi.org/10.14341/ osteo201223-8.

27. Belaya ZE, Iljin AV, Melnichenko GA, et al. Diagnostic performance of osteocalcin measurements in patients with endogenous Cushing's syndrome. Bonekey Rep. 2016;5:815. doi: https://doi.org/10.1038/bonekey.2016.42.

28. Mizokami A, Kawakubo-Yasukochi T, Hirata M. Osteocalcin and its endocrine functions. Biochem Pharmacol. 2017;132:1-8. doi: https://doi.org/10.1016/j.bcp.2017.02.001.

29. Гребенникова Т.А., Белая Ж.Е., Цориев Т.Т., и др. Эндокринная функция костной ткани. // Oстеопороз и остеопатии. - 2015. T.18. - №1. - C. 28-37. [Grebennikova TA, Belaya ZE, Tsoriev TT, et al. The Endocrine Function of the Bone Tissue. OSTEO. 2015;18(1):2837. (In Russ.)] doi: https://doi.org/10.14341/osteo2015128-37.

30. Marin L, Koivula M-K, Jukkola-Vuorinen A, et al. Comparison of total and intact aminoterminal propeptide of type 1 procollagen assays in patients with breast cancer with or without bone metastases. Ann Clin Biochem. 2011;48(Pt 5):447-451. doi: https://doi.org/10.1258/acb.2011.011040. 


\section{ИНФОРМАЦИЯ ОБ АВТОРАХ [AUTHORS INFO]}

*Цориев Тимур Тамерланович [Timur T. Tsoriev, MD]; адрес: Россия, 117036, Москва, улица Дм. Ульянова, д. 11 [address: 11 Dm. Ulyanova street, 117036 Moscow, Russia]; ORCID: http://orcid.org/0000-0001-9074-2291; eLibrary SPIN: 7234-2499; e-mail: timur.tsoriev@gmail.com.

Белая Жанна Евгеньевна, д.м.н. [Zhanna E. Belaya, MD, PhD]; e-mail: jannabelaya@gmail.com; ORCID: http://orcid.org/0000-0002-6674-6441; eLibrary SPIN: 4746-7173.

Рожинская Людмила Яковлевна, д.М.н., профессор [Lyudmila Y. Rozhinskaya, MD, PhD, Professor]; e-mail: rozh@endocrincentr.ru; ORCID: http://orcid.org/0000-0001-7041-0732; eLibrary SPIN: 5691-7775.

Никанкина Лариса Вячеславовна, К.М.н. [Larisa V. Nikankina, MD, PhD]; e-mail: larisanikan@rambler.ru; ORCID: http://orcid.org/0000-0001-8303-3825; eLibrary SPIN: 2794-0008.

\section{ИНФОРМАЦИЯ}

Рукопись получена: 02.07.2019. Одобрена к публикации: 25.07.2019.

\section{КАК ЦИТИРОВАТЬ:}

Цориев Т.Т., Белая Ж.Е., Рожинская Л.Я., Никанкина Л.В. Оценка диагностических возможностей коллагенового маркера костеобразования (P1NP) в сравнении с остеокальцином при болезни Иценко-Кушинга. // Остеопороз и остеопатии. — 2019. - Т. 22. — №1. — С.10-17. doi: https://doi.org/10.14341/osteo10266

\section{TO CITE THIS ARTICLE:}

Tsoriev TT, Belaya ZhE, Rozhinskaya LYa, Nikankina LV. Evaluation of diagnostic potential of the collagen osteogenesis marker (P1NP) compared with osteocalcin in Cushing's disease. Osteoporosis and bone diseases. 2019;22(1):10-17. doi: https://doi. org/10.14341/osteo10266 